\title{
Supporting Information \\ Overview of the Fiber Dynamics during Melt Blowing
}

Guangwu Sun 1,2,*, Wanli Han ${ }^{3}$, Yudong Wang ${ }^{4}$, Sanfa Xin ${ }^{1}$, Jingru Yang ${ }^{5,6}$, Fangdong Zou $^{5}$, Xinhou Wang ${ }^{5}$, Changfa Xiao ${ }^{1,7, *}$

${ }^{1}$ Fiber Materials Research Center, School of Textiles and Fashion, Shanghai University of Engineering Science, Shanghai, 201620, P. R. China

${ }^{2}$ Hainan Vocational University of Science and Technology, Haikou, Hainan Province, 571126, P.R. China

${ }^{3}$ Materials and Textile Engineering College, Jiaxing University, Jiaxing, Zhejiang Province, 314001, P. R. China

${ }^{4}$ College of Biological and Chemical engineering, Guangxi University of Science and Technology, Liuzhou, Guangxi Zhuang Autonomous

Region, 545006, P. R. China

${ }^{5}$ College of Textiles, Donghua University, 201620, Shanghai, P. R. China

${ }^{6}$ College of Textiles, Zhongyuan University of Technology, Zhengzhou, Henan, 450007, P.R. China

${ }^{7}$ State Key Laboratory of Separation Membranes and Membrane Processes, Tiangong University, Tianjin, 300387, P.R. China 
Table. S1 The Development of Fiber and Web Formation Theories

\begin{tabular}{|c|c|c|c|c|c|c|c|}
\hline $\begin{array}{l}\text { Principle } \\
\text { investigator }\end{array}$ & The $1^{\text {st }}$ author & $\begin{array}{l}\text { Dimension } \\
\text { of model }\end{array}$ & Constitutive equations & Correlation of parameter & Air flow data & $\begin{array}{l}\text { Die } \\
\text { types }\end{array}$ & Other innovations \\
\hline \multirow[t]{4}{*}{ Shambaugh } & Uyttendaele $^{10}$ & $1 \mathrm{D}$ & Newtonian fluid model & Viscosity-molecular weight & $\begin{array}{l}\text { Contact } \\
\text { measurement }\end{array}$ & $\begin{array}{l}\text { Annular } \\
\text { die }\end{array}$ & - \\
\hline & $\mathrm{Rao}^{39}$ & $2 \mathrm{D}$ & Newtonian fluid model & $\begin{array}{l}\text { Heat exchange coefficient-the } \\
\text { Nusselt number }\end{array}$ & $\begin{array}{l}\text { Contact } \\
\text { measurement }\end{array}$ & $\begin{array}{l}\text { Annular } \\
\text { die }\end{array}$ & - \\
\hline & $\operatorname{Marla}^{43,44}$ & $3 \mathrm{D}$ & $\begin{array}{l}\text { Newtonian fluid model } \\
\text { PTT model }\end{array}$ & Viscosity-temperature & $\begin{array}{l}\text { Contact } \\
\text { measurement }\end{array}$ & $\begin{array}{l}\text { Annular } \\
\text { die }\end{array}$ & - \\
\hline & Shambaugh $^{37}$ & $1 \mathrm{D}$ & $\begin{array}{l}\text { Newtonian fluid model } \\
\text { PTT model }\end{array}$ & Crystallinity-density & $\begin{array}{l}\text { CFD } \\
\text { software }\end{array}$ & $\begin{array}{l}\text { Annular } \\
\text { die }\end{array}$ & - \\
\hline \multirow[t]{2}{*}{$\begin{array}{l}\text { Huang and } \\
\text { Chen }\end{array}$} & Chen $^{25-28}$ & $1 \mathrm{D}$ & $\begin{array}{l}\text { Power-law model } \\
\text { Non-Newtonian fluid } \\
\text { model }\end{array}$ & $\begin{array}{l}\text { Temperature-density; } \\
\text { temperature-heat capacity }\end{array}$ & $\begin{array}{l}\text { CFD } \\
\text { software }\end{array}$ & Slot die & - \\
\hline & $\mathrm{Wu}^{49,50}$ & $1 \mathrm{D}$ & Maxwell model & - & $\begin{array}{l}\text { CFD } \\
\text { software }\end{array}$ & $\begin{array}{l}\text { Annular } \\
\text { die }\end{array}$ & Nonlinear surface tension \\
\hline Jarecki & Ziabicki ${ }^{32-36}$ & $1 \mathrm{D}$ & PPT model & $\begin{array}{l}\text { Temperature-density; } \\
\text { Crystallinity-density; } \\
\text { Temperature-heat capacity; } \\
\text { Heat exchange coefficient-the } \\
\text { Nusselt number; } \\
\text { Temperature-surface tension. }\end{array}$ & $\begin{array}{l}\text { CFD } \\
\text { software }\end{array}$ & & - \\
\hline Wang & Zeng $^{17}$ and & $3 \mathrm{D}$ & Maxwell model & - & CFD & Slot die & The molten \\
\hline
\end{tabular}




\begin{tabular}{|c|c|c|c|c|c|c|c|}
\hline & $\operatorname{Sun}^{18}$ & & & & software & & $\begin{array}{l}\text { modeled as a series of beads } \\
\text { connected by dashpots and } \\
\text { springs }\end{array}$ \\
\hline & $\operatorname{Han}^{55,56}$ & $3 \mathrm{D}$ & Standard linear solid & - & $\begin{array}{l}\text { CFD } \\
\text { software }\end{array}$ & Slot die & $\begin{array}{l}\text { The dashpots and springs are } \\
\text { arranged in different ways }\end{array}$ \\
\hline & $\operatorname{Sun}^{53,54,65-67}$ & $3 \mathrm{D}$ & Giesekus model & $\begin{array}{l}\text { Relaxation time-molecular } \\
\text { weight, temperature, } \\
\text { crystallinity; } \\
\text { Crystallinity-temperature, } \\
\text { external stress }\end{array}$ & $\begin{array}{l}\text { CFD } \\
\text { software }\end{array}$ & Slot die & $\begin{array}{l}\text { Multiple orifice dies and the } \\
\text { suction below the collector } \\
\text { were included; } \\
\text { Fiber diameter distribution, } \\
\text { weight distribution, and pore } \\
\text { size distribution were } \\
\text { predicted. }\end{array}$ \\
\hline \multirow[t]{3}{*}{ Yarin } & Sinha-Ray ${ }^{15,6}$ & Quasi-1D & UCM model & Viscosity-temperature; & CFD & - & Fiber diameter distribution, \\
\hline & $\begin{array}{l}9 \text { and } \\
\text { Yarin }^{16,68}\end{array}$ & & & Relaxation time-temperature & software & & $\begin{array}{l}\text { fiber orientation distribution, } \\
\text { weight distribution were } \\
\text { predicted. }\end{array}$ \\
\hline & Ghosal $^{60,70}$ & $3 \mathrm{D}$ & - & $\begin{array}{l}\text { Crystallinity-temperature, } \\
\text { external stress }\end{array}$ & - & - & $\begin{array}{l}\text { Porosity and thickness of the } \\
\text { web were simulated }\end{array}$ \\
\hline Bates & $\operatorname{Tan}^{30}$ & $1 \mathrm{D}$ & $\begin{array}{l}\text { UCM model; } \\
\text { PTT model }\end{array}$ & - & $\begin{array}{l}\text { Contact } \\
\text { measurement }\end{array}$ & Slot die & - \\
\hline Kumar & Zhou $^{58}$ & $1 \mathrm{D}$ & $\begin{array}{l}\text { UCM model; } \\
\text { PTT model } \\
\text { Newtonian fluid model } \\
\text { Giesekus model }\end{array}$ & - & $\begin{array}{l}\text { CFD } \\
\text { software }\end{array}$ & Slot die & - \\
\hline Wegener & Wieland $^{82,83}$ & $3 \mathrm{D}$ & UCM model & $\begin{array}{l}\text { Viscosity-temperature; } \\
\text { Relaxation time-temperature }\end{array}$ & $\begin{array}{l}\text { CFD } \\
\text { software }\end{array}$ & - & $\begin{array}{l}\text { The effects of turbulent } \\
\text { fluctuations are coupled. }\end{array}$ \\
\hline
\end{tabular}



Table. S2 The Development of Fiber Formation Mechanism Experiment

\begin{tabular}{|c|c|c|c|c|c|c|c|c|c|c|c|c|c|}
\hline $\begin{array}{l}\text { Principle } \\
\text { investigat }\end{array}$ & $\begin{array}{l}\text { The } \\
1^{\text {st }}\end{array}$ & $\begin{array}{r}\text { Measured } 1 \\
\text { measure }\end{array}$ & $\begin{array}{l}\text { rameters and } \\
\text { lent mode }\end{array}$ & $\begin{array}{c}\text { Employed } \\
\text { instruments } \\
\text { and }\end{array}$ & & Used & naterial & & & Proce & $\operatorname{sing} \mathrm{Cc}$ & aditions & \\
\hline & & $\begin{array}{c}\text { Online } \\
\text { measurem } \\
\text { ent }\end{array}$ & $\begin{array}{c}\text { Offline } \\
\text { measureme } \\
\text { nt }\end{array}$ & & Name & $\begin{array}{l}\text { Melt } \\
\text { Flow } \\
\text { Rate } \\
(\mathrm{g} / 10 \\
\text { min }) \\
\end{array}$ & $\begin{array}{c}\text { Mean } \\
\mathrm{M}_{\mathrm{w}}\end{array}$ & $\begin{array}{l}\text { Mean } \\
\mathrm{M}_{\mathrm{n}}\end{array}$ & $\begin{array}{c}\text { Air } \\
\text { velocity/ } \\
\text { Pressure }\end{array}$ & $\begin{array}{l}\text { Air } \\
\text { Temp- } \\
\text { eratur } \\
\text { e }\left({ }^{\circ} \mathrm{C}\right)\end{array}$ & $\begin{array}{l}\text { DCD } \\
(\mathrm{cm})\end{array}$ & $\begin{array}{c}\text { Die } \\
\text { temperat } \\
\text { ure } \\
\left({ }^{\circ} \mathrm{C}\right)\end{array}$ & $\begin{array}{c}\text { Collector } \\
\text { Speed }\end{array}$ \\
\hline \multirow{4}{*}{$\begin{array}{c}\text { Shambau } \\
\text { gh }\end{array}$} & $\begin{array}{l}\text { Uytte } \\
\text { ndael } \\
\mathrm{e}^{10}\end{array}$ & $\begin{array}{c}\text { Fiber } \\
\text { vibration; } \\
\text { Fiber } \\
\text { diameter }\end{array}$ & $\begin{array}{c}\text { Crystallinit } \\
y\end{array}$ & $\begin{array}{c}\text { High-speed } \\
\text { flash; } \\
\text { Digital } \\
\text { camera; } \\
\text { DSC }\end{array}$ & PP & 35 & 120,000 & 30,000 & $\begin{array}{c}110.28 \mathrm{~m} / \\
\mathrm{s} \\
57.93 \mathrm{~m} / \mathrm{s}\end{array}$ & $\begin{array}{l}368 \\
385\end{array}$ & - & 400 & - \\
\hline & $\begin{array}{c}\text { Kayse } \\
\mathrm{r}^{111}\end{array}$ & - & $\begin{array}{c}\text { Fiber } \\
\text { diameter }\end{array}$ & $\begin{array}{c}\text { Optical } \\
\text { microscopy }\end{array}$ & $\begin{array}{c}\mathrm{PP} \\
\mathrm{PP} \\
\mathrm{PE}\end{array}$ & $\begin{array}{c}54 \\
93 \\
125\end{array}$ & $\begin{array}{c}157,000 \\
119,000 \\
-\end{array}$ & $\begin{array}{c}40,700 \\
47,800 \\
-\end{array}$ & $\begin{array}{l}- \\
- \\
-\end{array}$ & $\begin{array}{l}- \\
- \\
-\end{array}$ & 30 & $\begin{array}{c}350,400 \\
350 \\
325\end{array}$ & - \\
\hline & $\mathrm{Wu}^{107}$ & $\begin{array}{c}\text { Fiber } \\
\text { velocity }\end{array}$ & - & LDV & $\begin{array}{c}\mathrm{PP} \\
\mathrm{PP}(95) / \\
\operatorname{PET}(5)\end{array}$ & 54 & 157,000 & 40,700 & $\begin{array}{c}90 \mathrm{~m} / \mathrm{s} \\
\sim \\
118 \mathrm{~m} / \mathrm{s}\end{array}$ & 395 & - & 400 & - \\
\hline & $\mathrm{Rao}^{39}$ & $\begin{array}{c}\text { Fiber } \\
\text { vibration; } \\
\text { Fiber }\end{array}$ & - & $\begin{array}{c}\text { High-speed } \\
\text { flash; } \\
\text { Digital }\end{array}$ & PP & 75 & - & - & $\begin{array}{c}62 \mathrm{~m} / \mathrm{s} \\
110 \mathrm{~m} / \mathrm{s} \\
\sim \\
300 \mathrm{~m} / \mathrm{s}\end{array}$ & 368 & - & 305 & - \\
\hline
\end{tabular}




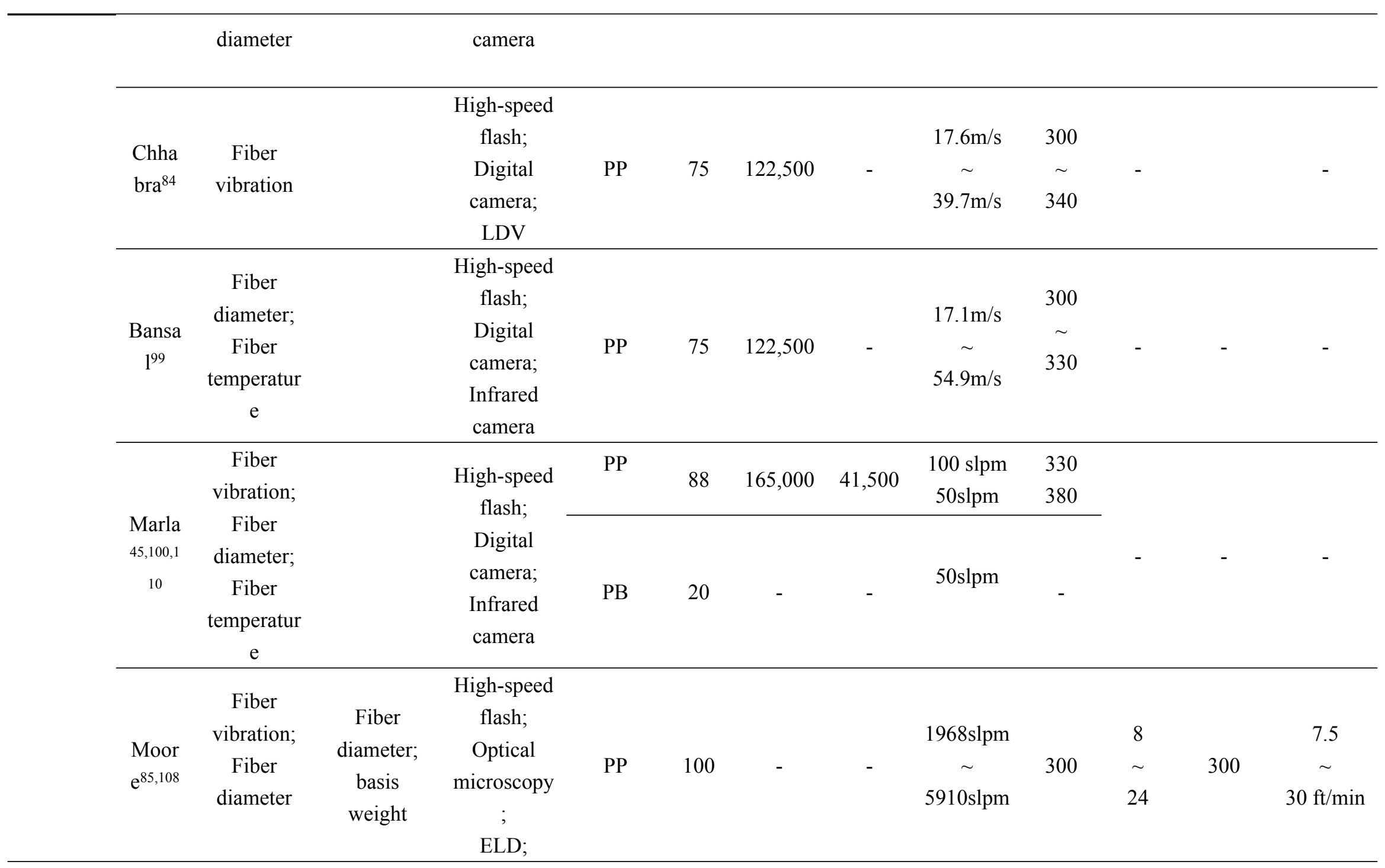




\begin{tabular}{|c|c|c|c|c|c|c|c|c|c|c|c|c|c|}
\hline & & & & $\begin{array}{c}\text { SEM; } \\
\text { Image } \\
\text { analysis }\end{array}$ & & & & & & & & & \\
\hline & $\begin{array}{l}\text { Beard } \\
86\end{array}$ & $\begin{array}{l}\text { Fiber } \\
\text { vibration; }\end{array}$ & & $\begin{array}{l}\text { High speed } \\
\text { camera }\end{array}$ & PP & 88 & 165,000 & 41,500 & 150 slpm & 368 & - & 310 & - \\
\hline Zeng & $\begin{array}{l}\mathrm{Xie}^{51} \\
87-90,10 \\
3\end{array}$ & $\begin{array}{l}\text { Fiber } \\
\text { vibration; } \\
\text { Fiber } \\
\text { diameter }\end{array}$ & & $\begin{array}{l}\text { High-speed } \\
\text { camera }\end{array}$ & PP & 650 & - & - & $\begin{array}{l}0.5-1.25 \\
\text { atm }\end{array}$ & 260 & - & - & - \\
\hline \multirow[t]{3}{*}{$\begin{array}{l}\text { Yarin and } \\
\text { Pourdeyh } \\
\text { imi }\end{array}$} & $\begin{array}{l}\text { Sinha } \\
- \text { Ray }^{1} \\
5\end{array}$ & $\begin{array}{l}\text { Thread } \\
\text { vibration; }\end{array}$ & & $\begin{array}{l}\text { Digital } \\
\text { camera }\end{array}$ & $\begin{array}{l}\text { Sewing } \\
\text { thread }\end{array}$ & - & - & - & 35 bar & - & - & - & - \\
\hline & $\begin{array}{l}\text { Hassa } \\
\mathrm{n}^{124}\end{array}$ & & $\begin{array}{l}\text { Fiber } \\
\text { diameter }\end{array}$ & SEM & PP & 1800 & - & - & - & 280 & $\begin{array}{l}19 \\
14\end{array}$ & 240 & $\begin{array}{l}14.85 \\
\mathrm{~m} / \mathrm{min}\end{array}$ \\
\hline & $\begin{array}{l}\text { Ghosa } \\
1^{60}\end{array}$ & & $\begin{array}{l}\text { Fiber } \\
\text { crystallinity }\end{array}$ & DSC & PP & - & - & - & - & $\begin{array}{l}255-2 \\
62\end{array}$ & $\begin{array}{l}15 \\
\sim \\
30 \\
\end{array}$ & & $\begin{array}{l}13.2 \\
39.8\end{array}$ \\
\hline Bresee & $\begin{array}{l}\text { Brese } \\
\mathrm{e}^{96-98,1} \\
40,144,1 \\
51-154\end{array}$ & $\begin{array}{l}\text { Fiber } \\
\text { vibration; } \\
\text { Fiber } \\
\text { diameter; } \\
\text { Fiber } \\
\text { speed }\end{array}$ & $\begin{array}{l}\text { Fiber } \\
\text { crystallinity } \\
\text {; basis } \\
\text { weight; } \\
\text { shot; fiber } \\
\text { diameter; } \\
\text { pore size; } \\
\text { Fiber }\end{array}$ & $\begin{array}{l}\text { High-speed } \\
\text { camera; } \\
\text { WAXD; } \\
\text { SAXS; } \\
\text { Image } \\
\text { analysis }\end{array}$ & PP & 400 & - & - & $\begin{array}{l}370 \\
\mathrm{ft}^{3} / \mathrm{min} \\
\sim \\
700 \\
\mathrm{ft}^{3} / \mathrm{min}\end{array}$ & - & $\begin{array}{l}22 \\
\sim \\
40 ;\end{array}$ & $\begin{array}{l}190 \\
\sim \\
260\end{array}$ & - \\
\hline
\end{tabular}


orientation

\begin{tabular}{|c|c|c|c|c|c|c|c|c|c|c|c|c|c|}
\hline & Yin $^{101}$ & $\begin{array}{l}\text { Fiber } \\
\text { vibration; } \\
\text { Fiber } \\
\text { diameter; } \\
\text { Fiber } \\
\text { temperatur } \\
\text { e }\end{array}$ & $\begin{array}{l}\text { Fiber } \\
\text { birefringen } \\
\text { ce; Fiber } \\
\text { crystallinity }\end{array}$ & $\begin{array}{l}\text { High-speed } \\
\text { camera; } \\
\text { Infrared } \\
\text { thermomete } \\
\text { r; } \\
\text { Polarized } \\
\text { optical } \\
\text { microscopy } \\
\text {; } \\
\text { DSC; } \\
\text { LDV; }\end{array}$ & PP & 1259 & - & - & - & - & 90 & - & - \\
\hline $\mathrm{Ke}$ & $\begin{array}{l}\text { Wang } \\
112\end{array}$ & - & $\begin{array}{l}\text { Fiber } \\
\text { diameter }\end{array}$ & $\begin{array}{l}\text { Optical } \\
\text { microscopy }\end{array}$ & $\begin{array}{l}\text { Copolya } \\
\text { mide }\end{array}$ & - & - & - & $\begin{array}{l}0.18 \mathrm{MPa} \\
\sim \\
0.38 \mathrm{MPa}\end{array}$ & 292 & $\begin{array}{l}85 \\
\sim \\
120\end{array}$ & 170 & - \\
\hline Lee & $\begin{array}{l}\text { Lee }^{113} \\
, 120\end{array}$ & - & $\begin{array}{l}\text { Fiber } \\
\text { diameter, } \\
\text { Pore size; } \\
\text { fiber } \\
\text { orientation }\end{array}$ & $\begin{array}{l}\text { Optical } \\
\text { microscopy } \\
; \\
\text { SEM; } \\
\text { Coulter } \\
\text { Porometer }\end{array}$ & $\begin{array}{l}\text { Thermo } \\
\text { plastic } \\
\text { polyuret } \\
\text { hanes; } \\
\text { PP }\end{array}$ & 700 & 93,000 & 27,000 & $\begin{array}{l}160 \\
\mathrm{ft}^{3} / \min \\
\sim \\
370 \\
\mathrm{ft}^{3} / \min \end{array}$ & - & $\begin{array}{l}16 \\
\sim \\
61\end{array}$ & $\begin{array}{l}207 \\
\sim \\
246\end{array}$ & $30 \mathrm{rpm}$ \\
\hline $\begin{array}{l}\text { Huang } \\
\text { and Chen }\end{array}$ & $\begin{array}{l}\text { Chen }^{2} \\
7,28,117\end{array}$ & - & $\begin{array}{l}\text { Fiber } \\
\text { diameter }\end{array}$ & $\begin{array}{l}\text { Three-dime } \\
\text { nsional } \\
\text { video }\end{array}$ & PBT & 62 & - & - & $\begin{array}{l}91 \mathrm{~m} / \mathrm{s} \\
\sim \\
311 \mathrm{~m} / \mathrm{s}\end{array}$ & $\begin{array}{l}280 \\
320\end{array}$ & $\begin{array}{l}10 \\
\sim \\
14\end{array}$ & - & - \\
\hline
\end{tabular}




\begin{tabular}{|c|c|c|c|c|c|c|c|c|c|c|c|c|c|}
\hline & & & & microscope & PP & 54 & - & - & $168 \mathrm{~m} / \mathrm{s}$ & 310 & & - & - \\
\hline & $\mathrm{Wu}^{118}$ & - & Fiber & Three-dime & Polyacti & 12 & - & - & $247 \mathrm{~m} / \mathrm{s}$ & 220 & 90 & - & - \\
\hline & & & diameter & nsional & $\mathrm{c}$ & & & & $\sim$ & 320 & $\sim$ & & \\
\hline & & & & video & & & & & $273 \mathrm{~m} / \mathrm{s}$ & & 110 & & \\
\hline & & & & microscope & & & & & & & & & \\
\hline \multirow[t]{10}{*}{ Bates } & $\operatorname{Tan}^{30}$ & - & Fiber & SEM & PS & - & - & 3,000 & - & 165 & 55 & - & - \\
\hline & & & diameter & & & & & & & 180 & & & \\
\hline & & & & & & & & 610,00 & & & & & \\
\hline & & & & & & & & 0 & & & & & \\
\hline & Elliso & - & Fiber & SEM & PS & - & 48,800 & 2,100 & - & 180 & 55 & & - \\
\hline & $\mathrm{n}^{121}$ & & diameter & & & & & & & $\sim$ & & - & \\
\hline & & & & & & & & & & 280 & & & \\
\hline & & & & & PP & 1500 & 59,000 & 10,600 & - & 180 & & & - \\
\hline & & & & & & & & & & 220 & & - & \\
\hline & & & & & PBT & 250 & - & - & - & 265 & & - & - \\
\hline \multirow[t]{9}{*}{ Wang } & $\operatorname{Han}^{12}$ & - & Fiber & SEM; & PP & 1550 & - & - & $34 \mathrm{KPa}$ & - & - & 230 & - \\
\hline & 7 & & diameter; & Capillary & & & & & $\sim$ & & & & \\
\hline & & & Pore size & flow & & & & & $103 \mathrm{KPa}$ & & & & \\
\hline & & & & porometer & & & & & & & & & \\
\hline & $\operatorname{Sun}^{57}$ & - & Basis & Image & PP & 15 & - & - & $150 \mathrm{KPa}$ & 150 & 50 & - & $4 \mathrm{~cm} / \mathrm{s}$ \\
\hline & 67 & & weight; & analysis; & & & & & $\sim$ & $\sim$ & $\sim$ & & $\sim$ \\
\hline & & & pore size & Capillary & & & & & $400 \mathrm{KPa}$ & 270 & 130 & & $12 \mathrm{~cm} / \mathrm{s}$ \\
\hline & & & & flow & & & & & & & & & \\
\hline & & & & porometer & & & & & & & & & \\
\hline \multirow[t]{2}{*}{ Zatloukal } & Drabe & - & Fiber & SEM & PP & 450 & 75,850 & 17,200 & & 270 & 20 & - & $4 \mathrm{~m} / \mathrm{min}$ \\
\hline & $\mathrm{k}^{134-13}$ & & diameter & & & 1200 & 56,250 & 14,250 & & & & & \\
\hline
\end{tabular}




\begin{tabular}{|c|c|c|c|c|c|c|c|c|c|c|c|c|c|}
\hline & 6 & & & & & & & & & & & & \\
\hline \multirow[t]{22}{*}{ Bhat } & Hoda $^{1}$ & - & Fiber & SEM & PP & 1200 & - & - & $35 \mathrm{KPa}$ & 170 & - & 190 & 20rpm \\
\hline & & & diameter & & & & & & $\sim$ & $\sim$ & & $\sim$ & $\sim$ \\
\hline & & & & & & & & & $103 \mathrm{KPa}$ & 210 & & 230 & 50rpm \\
\hline & Hegd & - & Fiber & Polarized & PP & 1500 & & & $21 \mathrm{kN} / \mathrm{m}^{2}$ & & 38 & 216 & $12 \mathrm{~m} / \mathrm{min}$ \\
\hline & $\mathrm{e}^{122}$ & & diameter; & optical & & & & & $41 \mathrm{kN} / \mathrm{m}^{2}$ & & & $\sim$ & \\
\hline & & & Thickness; & microscope & & & & & & & & 288 & \\
\hline & & & Fiber & & & & & & & & & & \\
\hline & & & crystallinity & Thickness & & & & & & & & & \\
\hline & & & and crystal & tester; & & & & & & & & & \\
\hline & & & size; pore & $\mathrm{DSC}$ & & & & & & & & & \\
\hline & & & & WAXD; & & & & & & & & & \\
\hline & & & & Transmissi & & & & & & & & & \\
\hline & & & & on electron & & & & & & & & & \\
\hline & & & & microscopy & & & & & & & & & \\
\hline & Uppal & - & Fiber & SEM; & PP & - & - & - & $69 \mathrm{KPa}$ & 255 & 2.5 & 240 & 0.86 \\
\hline & 123 & & diameter; & Capillary & & & & & $\sim$ & & $\sim$ & & $\mathrm{mpm}$ \\
\hline & & & pore size & flow & & & & & $138 \mathrm{KPa}$ & & 3.5 & & \\
\hline & & & & porometer & & & & & & & & & \\
\hline & Yesil $^{1}$ & - & Fiber & SEM; & PE & 155 & - & - & $20 \mathrm{KPa}$ & 240 & 15 & 231 & $5.8 \mathrm{~m} / \mathrm{mi}$ \\
\hline & 30,156 & & diameter; & Capillary & & & & & $\sim$ & 255 & $\sim$ & & $\mathrm{n}$ \\
\hline & & & pore size & flow & & & & & $70 \mathrm{KPa}$ & & 35 & & \\
\hline & & & & porometer & & & & & & & & & \\
\hline \multirow[t]{3}{*}{ Formoso } & Form & Fiber & - & High-speed & Ethylen & - & - & - & $2.8 \mathrm{~L} / \mathrm{min}$ & 200 & - & - & - \\
\hline & oso $^{92,9}$ & vibration; & & camera & $\mathrm{e}$ & & & & /inch & & & & \\
\hline & 4 & Fiber & & & copolym & & & & $\sim$ & & & & \\
\hline
\end{tabular}


$\mathrm{n} /$ inch

Abbreviation:

$\mathrm{PET}=$ polyester

$\mathrm{Slpm}=$ stard liter per minute

$\mathrm{PS}=$ Polystyrene

$\mathrm{PBT}=$ Polybutylece terephthalate

$\mathrm{PB}=$ Polybutylene

$\mathrm{PE}=$ Polyethylene 